\title{
About the Exactness of the Linear Response Theory
}

\author{
D. Goderis ${ }^{\star}$, A. Verbeure and P. Vets ${ }^{\star \star}$ \\ Instituut voor Theoretische Fysica, Universiteit Leuven, B-3001 Leuven, Belgium
}

Received January 29, 1990

\begin{abstract}
For quantum lattice systems with finite range potentials and integrable space clustering, we prove the linearity of the response theory when dealing with fluctuation observables.
\end{abstract}

\section{Introduction}

In statistical mechanics an equilibrium state of a finite system is given by a Gibbs state. In the thermodynamic limit, i.e. for infinite systems, the equilibrium states are described by states satisfying the KMS-condition.

A problem is to understand the occurrence of equilibrium from a dynamical point of view. This is tackled in different ways. One can study the problem by considering the system as part of a larger one. This leads to open system considerations, where topics like the master equation are widely studied (see e.g. [1]). Some aspects of this theory have been made rigorous by several authors in the last decade [2]. Another way to approach the problem is to consider small dynamical perturbations of the system and to observe the effect on the system. Technically one considers a straightforward Dyson expansion of the perturbed dynamics in terms of the unperturbed one. It is often argued that when the perturbation is small and when near to equilibrium, one can limit the study of the response to the first order term in the expansion. This is the basis of the well known "linear response theory" of Kubo $[3,4]$. Some aspects of this linear response theory as developed in [3] and [5] have been proved rigorously in [6,7]. However a lot had to be done to get a complete theory of linear response. This theory consists in a simplistic first order perturbational calculation for which there is not a general theory for treating systematically the higher order terms. On the other hand the linear response actually observed in macroscopic systems has a physical significant range of validity.

$\star$ Onderzoeker IIKW, Belgium

$\star \star$ Onderzoeker IIKW, Belgium 
From a theoretical point of view the most severe criticism of the linear response theory was expressed by Van Kampen [8]. He points out that the dynamics of a system can be very sensitive even to small perturbations, such that a perturbational calculation becomes impossible. He claims that microscopic linearity and macroscopic linearity are totally different things. He puts forward that the latter can only be understood by a kinetic approach. Kubo et al. reply to this criticism [4]. They answer that working near to equilibrium might save the perturbational approach. However this statement remained without proof. A second argument is based on the stochastization entering through the thermodynamic limit. They claim that this again is difficult to prove.

Our present work has to be considered as a contribution to the understanding of the validity of the response theory being linear.

Already in [9] it is rigorously proved that the response is linear if one considers the response of fluctuation observables. This result was a first step, but rather weak in the sense that the proof holds only for small values of the time parameter. One expects it to hold in particular for large times. In this paper we are able to sharpen this result in different directions, using the theory of macrofluctuations for quantum systems, derived on the basis of the central limit theory. The algebra of fluctuations is a representation of the canonical commutation relations induced by a generalized free state. We learned also that the natural conservative time evolution of the system induces a nontrivial dynamics on the fluctuations [12].

Here we consider a dynamics perturbed by a fluctuation and prove the existence of a perturbed dynamics for the algebra of fluctuations. We work out all this for spin systems with finite range interactions in a state which has integrable space clustering. In particular we prove that this macrodynamics is linear in the perturbation. We prove that the linear response theory becomes exact for all values of the time on the macroscopic level. We remark at this point that it is not necessary to start with a system at equilibrium. The result is a mere consequence of coarse graining due to the central limits. This result shows that microscopic and macroscopic linearity are different phenomena which can appear simultaneously but at different levels.

Moreover if the microsystem is in equilibrium, we construct the equilibrium macrostate of the perturbed dynamics and recover the correct response and relaxation functions in terms of the Duhamel two-point function. This rigorous treatment should clarify the controversy about the linearity of the response theory and reveal its origins.

\section{The Model and Preliminaries}

We develop the theory for systems which are defined on a $v$-dimensional lattice $\mathbb{Z}^{v}$ and which have a quasi-local structure [10]. Let $\mathscr{D}\left(\mathbb{Z}^{v}\right)$ be the directed set of finite subsets of $\mathbb{Z}^{v}$, where the direction is the inclusion. With each $x \in \mathbb{Z}^{v}$ we associate the algebra $\mathscr{A}_{x}$, a copy of a matrix algebra $M_{N}$ of $N \times N$ matrices. For all $\Lambda \in \mathscr{D}\left(\mathbb{Z}^{v}\right)$, consider the tensor product $\mathscr{A}_{\Lambda}=\bigotimes \mathscr{A}_{x}$. The family $\mathscr{A}_{\Lambda}, \Lambda \in \mathscr{D}\left(\mathbb{Z}^{v}\right)$ has the usual properties of locality and isotony: $x \in \Lambda$

$$
\begin{array}{ccc}
{\left[\mathscr{A}_{\Lambda_{1}}, \mathscr{A}_{\Lambda_{2}}\right]=0} & \text { if } & \Lambda_{1} \cap \Lambda_{2}=\emptyset, \\
\mathscr{A}_{\Lambda_{1}} \subseteq \mathscr{A}_{\Lambda_{2}} & \text { if } & \Lambda_{1} \subseteq \Lambda_{2} .
\end{array}
$$


Denote by $\mathscr{A}_{L}$ all local observables

$$
A_{L}=\bigcup_{\Lambda \in \mathscr{D}\left(\mathbb{Z}^{v}\right)} \mathscr{A}_{\Lambda}
$$

The norm closure $\mathscr{B}$ of $\mathscr{A}_{L}$ is again a $C^{*}$-algebra:

$$
\mathscr{B}=\overline{\mathscr{A}}_{L}=\overline{\bigcup_{\Lambda \in \mathscr{D}\left(\mathbb{Z}^{v}\right)} \mathscr{A}_{\Lambda}}
$$

and considered as the algebra of quasi-local observables of our system.

The group $\mathbb{Z}^{v}$ of space-translations of the lattice acts as a group of *-automorphisms on $\mathscr{B}$ by:

$$
\tau_{x}: A \in \mathscr{A}_{\Lambda} \rightarrow \tau_{x}(A) \in \mathscr{A}_{\Lambda+x} ; \quad x \in \mathbb{Z}^{\nu} .
$$

The dynamics of our system is determined in the usual way by the local Hamiltonians

$$
H_{\Lambda}=\sum_{X \subseteq \Lambda} \phi(X) ; \quad \Lambda \in \mathscr{D}\left(\mathbb{Z}^{\nu}\right)
$$

with

$$
\begin{gathered}
\phi(X) \in \mathscr{A}_{X} \text { for } X \in \mathscr{D}\left(\mathbb{Z}^{\nu}\right), \\
\tau_{x} \phi(X)=\phi(X+x) ; \quad x \in \mathbb{Z}^{\nu},
\end{gathered}
$$

and such that, there exists $\lambda>0$ :

$$
\|\phi\|_{\lambda} \equiv \sum_{0 \in X}|X| N^{2|X|} e^{\lambda d(X)}\|\phi(X)\|<\infty,
$$

where $d(X)=\sup _{x, y \in X}|x-y|$, is the diameter of the set $X$ and $|X|$ is the number of elements in $X$. From Sect. 3 on, we suppose that $\phi$ has a finite range, so that condition (2.5) is trivially satisfied.

For $\Lambda \in \mathscr{D}\left(\mathbb{Z}^{v}\right)$, the local dynamics $\alpha_{t}^{\Lambda}$ is given by

$$
\begin{aligned}
\alpha_{t}^{\Lambda}: \mathscr{A}_{\Lambda} & \rightarrow \mathscr{A}_{\Lambda} \\
\alpha_{t}^{\Lambda}(A) & =e^{i t H_{\Lambda}} A e^{-i t H_{\Lambda}}, \quad A \in \mathscr{A}_{\Lambda} .
\end{aligned}
$$

From (2.5) it follows that the global dynamics $\alpha_{t}$ of $\mathscr{B}$ exists as the following norm limit:

$$
\alpha_{t}=\lim _{\Lambda} \alpha_{t}^{\Lambda}
$$

and one has the following estimate [10, Theorem 6.2.11]:

$$
\left\|\alpha_{t}(A)-\alpha_{t}^{\Lambda}(A)\right\| \leqq\|A\|\left|\Lambda_{0}\right|\left(e^{\left.2|t||| \phi\right|_{\lambda}}-1\right) \sum_{x \in \Lambda^{c}} e^{-\lambda|x|_{0}} .
$$

where

$$
|x|_{0}=\min _{y \in \Lambda_{0}}|x-y| ; \quad A \in \mathscr{A}_{\Lambda_{0}} .
$$

By (2.4) one also has $\left[\alpha_{t}, \tau_{x}\right]=0$ for $t \in \mathbb{R}, x \in \mathbb{Z}^{v}$.

Finally we consider the $C^{*}$-system $\left(\mathscr{B}, \alpha_{t}, \omega\right)$, where $\omega$ is a state of $\mathscr{B}$ which is space and time translation invariant, i.e. $\omega^{\circ} \tau_{x}=\omega$ for all $x \in \mathbb{Z}^{v}$ and $\omega^{\circ} \alpha_{t}=\omega$ for $t \in \mathbb{R}$. 
Furthermore we assume that the state has the following space-clustering property: let

$$
\alpha_{\omega}(d)=\sup _{\Lambda, \tilde{\Lambda}} \sup _{\substack{A \in \mathscr{A}_{\Lambda} \\ B \in \mathscr{A}_{\tilde{\Lambda}}}}\left\{\frac{1}{\|A\|\|B\|}|\omega(A B)-\omega(A) \omega(B)| ; d \leqq d(\Lambda, \tilde{\Lambda})\right\}
$$

we suppose that

$$
\sum_{x \in \mathbb{Z}^{v}} \alpha_{\omega}(|x|)<\infty
$$

Remark that this space-factorization or clustering condition is of the same type as in [11], where the asymptotic orbits of non-interacting Fermi particles are studied. They assume that the cluster function $\alpha_{\omega}$ is a bit stronger than of the logarithmic type, we assume the $L^{1}$-type in (2.8). We can derive the results of this paper under the weaker condition, namely: there exists $\delta>0$ such that

$$
\lim _{N \rightarrow \infty} \sqrt{N} \alpha_{\omega}^{N}\left(N^{(1 / 2 v)-\delta}\right)=0
$$

where

$$
\alpha_{\omega}^{N}(d)=\sup _{\substack{\Lambda, \tilde{\Lambda} \\ A \in \mathscr{A}_{\Lambda} \\ B \in \mathscr{A}_{\tilde{\Lambda}}}}\left\{\frac{1}{\|A\|\|B\|}|\omega(A B)-\omega(A) \omega(B)| ; d \leqq d(\Lambda, \tilde{\Lambda}), \max (|\Lambda|,|\tilde{\Lambda}|) \leqq N\right\}
$$

One readily checks that (2.8) implies (2.9). However for technical convenience we stick to the condition (2.8).

Remark that the function $\alpha_{\omega}$ has the following immediate properties:

$$
2 \geqq \alpha_{\omega} \geqq 0
$$

and monotonically decreasing

$$
\alpha_{\omega}(d) \leqq \alpha_{\omega}\left(d^{\prime}\right) \text { for } \quad d^{\prime} \leqq d .
$$

Denote by $\Lambda_{n}$ the cube centered around the origin with edges of length $2 n+1$. For any element $A \in \mathscr{A}_{L, s a}$, the selfadjoint elements of $\mathscr{A}_{L}$, consider the local fluctuation $\tilde{A}_{n}$ of $A$ :

$$
\tilde{A}_{n}=\frac{1}{\left|\Lambda_{n}\right|^{1 / 2}} \sum_{x \in \Lambda_{n}}\left(\tau_{x} A-\omega(A)\right)
$$

In [12, Theorem 3.2] we prove that under the condition (2.8) the central limits exist: $A, B \in \mathscr{A}_{L, s a}$

$$
\lim _{n \rightarrow \infty} \omega\left(e^{i \tilde{A}_{n}} e^{i \tilde{B}_{n}}\right)=\exp \left\{-\frac{1}{2} S_{\omega}(A+B, A+B)-\frac{i}{2} \sigma_{\omega}(A, B)\right\},
$$

where

$$
\begin{aligned}
& s_{\omega}(A, B)=\operatorname{Re} \sum_{x}\left(\omega\left(A \tau_{x} B\right)-\omega(A) \omega(B)\right) \\
& \sigma_{\omega}(A, B)=-\sum_{x} \omega\left(\left[A, \tau_{x} B\right]\right) .
\end{aligned}
$$


We introduce the algebra of normal fluctuations of the system $(\mathscr{B}, \omega)$. Consider the symplectic space $\left(\mathscr{A}_{L, s a}, \sigma_{\omega}\right)$ and the $\mathrm{CCR}-C^{*}$-algebra $W\left(\mathscr{A}_{L, s a}, \sigma_{\omega}\right)$ generated by the Weyl operators $\left\{W(A) \mid A \in \mathscr{A}_{L, s a}\right\}$ satisfying the product rule

$$
W(A) W(B)=W(A+B) \exp -\frac{i}{2} \sigma_{\omega}(A, B) .
$$

Moreover, by [12, Theorem 3.3], the central limit theorem (2.11) fixes a representation of this CCR-algebra induced by a quasi-free state $\tilde{\omega}$ of the CCR $-C^{*}$-algebra defined by the relation

$$
\tilde{\omega}(W(A))=\lim _{n} \omega\left(e^{i \tilde{A}_{n}}\right)=e^{-(1 / 2) s_{\omega}(A, A)} .
$$

Also, if $\gamma$ is a $*$-automorphism of $\mathscr{B}$ leaving $\mathscr{A}_{L}$ invariant, commuting with the space translations and leaving the state $\omega$ invariant then $\tilde{\gamma}$ given by

$$
\tilde{\gamma}(W(A))=W(\gamma(A)), \quad A \in \mathscr{A}_{L, s a}
$$

defines a quasi-free *-automorphism of the CCR $-C^{*}$-algebra $W\left(\mathscr{A}_{L, s a}, \sigma_{\omega}\right)$.

The quasi-free state $\tilde{\omega}(2.15)$ has a GNS-representation $(\tilde{\pi}, \tilde{\mathscr{H}}, \tilde{\Omega})$ and a von Neumann algebra

$$
\tilde{\mathscr{M}}=\tilde{\pi}\left(W\left(\mathscr{A}_{L, s a}, \sigma_{\omega}\right)\right)^{\prime \prime}
$$

This algebra will be called the algebra of normal (macroscopic) fluctuations.

As the map $\lambda \in \mathbb{R} \rightarrow \tilde{\pi}(W(\lambda A))$ is strongly continuous for all $A$, Stone's theorem yields the existence of Boson fields $b(A)$ such that

$$
\tilde{\pi}(W(A))=\exp i b(A)
$$

and the Bose commutation relations:

$$
[b(A), b(B)]=i \sigma_{\omega}(A, B) .
$$

Remark that by (2.15),(2.17) and (2.18), one can identify the macroscopic fluctuations in the state $\omega$ with the Boson fields:

$$
\text { central- } \lim _{n \rightarrow \infty} \tilde{A}_{n}=b(A) \text {. }
$$

Clearly the map

$$
A \in \mathscr{A}_{L, s a} \rightarrow b(A)
$$

is not injective, e.g. $b\left(\tau_{x} A\right)=b(A)$ for all $x \in \mathbb{Z}^{v}$ and $A \in \mathscr{A}_{L, s a}$. This is a mathematical description of the physical notion of coarse graining [12].

Consider now $\mathscr{A}_{L}$ equipped with the sesquilinear form

$$
\begin{aligned}
\langle A, B\rangle_{s} & =s_{\omega}(A, B)+\frac{i}{2} \sigma_{\omega}(A, B) \\
& =\sum_{x \in \mathbb{Z}^{v}} \omega\left(A^{*} \tau_{x} B\right)-\omega\left(A^{*}\right) \omega(B) .
\end{aligned}
$$

Dividing $\mathscr{A}_{L}$ by the kernel of this sesquilinear form and completing the quotient one gets a complex Hilbert space $\mathscr{K}_{\omega}$ with scalar product (2.20). We denote by $\mathscr{K}_{\omega}^{\mathrm{Re}}$ the real subspace of $\mathscr{K}_{\omega}$ generated by $\mathscr{A}_{L, s a}$. 
Now we consider the extended CCR $-C^{*}$-algebra $W\left(\mathscr{K}_{\omega}^{\mathrm{Re}}, \sigma_{\omega}\right)$ and check on the basis of [13] Proposition 5, [12] Proposition 3.5 that

$$
\tilde{\mathscr{M}}=\tilde{\pi}\left(W\left(\mathscr{K}_{\omega}^{\mathrm{Re}}, \sigma_{\omega}\right)\right)^{\prime \prime},
$$

where $\tilde{\mathscr{M}}$ is given in (2.16). This settles the mathematical description of the fluctuation algebra $\tilde{\mathscr{M}}$ of which we remind that it is generated by the bounded continuous functions of the fluctuation fields $\left\{b(A) \mid A \in \mathscr{K}_{\omega}^{\mathrm{Re}}\right\}$.

The microscopic dynamics $\alpha_{t}$ can now be transported to a dynamics $\tilde{\alpha}_{t}$ of $\tilde{\mathscr{M}}[12$, Theorem 4.5].

First consider the time evolution on the space $\mathscr{K}_{\omega}$. One checks that the map $U_{t}: \mathscr{A}_{L} \rightarrow \mathscr{K}_{\omega}: U_{t} A=\alpha_{t} A$ extends to a unitary operator for all $t \in \mathbb{R}$. On the basis of the conditions (2.5) and (2.8), $\tilde{\alpha}_{t}$ defined on $W\left(\mathscr{K}_{\omega}^{\mathrm{Re}}, \sigma_{\omega}\right)$ :

$$
\tilde{\alpha}_{t} W(A)=W\left(U_{t} A\right), \quad A \in \mathscr{K}_{\omega}^{\mathrm{Re}}
$$

extends to a weakly continuous one-parameter group of *-automorphisms of $\tilde{\mathscr{M}}$.

Furthermore if $\omega$ is a $\left(\alpha_{t}, \beta\right)-$ KMS state of the micro-algebra of observables $\mathscr{B}$, then $\tilde{\omega}(2.15)$ is a $\left(\tilde{\alpha}_{t}, \beta\right)-\mathrm{KMS}$ state of the macro-algebra of observables $\tilde{\mathscr{M}}$.

We recapitulate the results of [12], the transition from a micro-system to a

\begin{tabular}{|c|c|c|}
\hline MICRO-SYSTEM & $\longrightarrow$ & MACRO-SYSTEM \\
\hline & central limit & \\
\hline$\left(\mathscr{A}_{L}, \omega\right)$ & implies & $(\tilde{\mathscr{M}}, \tilde{\omega})$ \\
\hline$A \in \mathscr{K}_{\omega}^{\mathrm{Re}}$ & implies & $\begin{array}{l}b(A), \text { boson field } \\
\text { affiliated to } \tilde{\mathscr{M}}\end{array}$ \\
\hline$\alpha_{t}$ & implies & $\tilde{\alpha}_{t}$ \\
\hline$\alpha_{t} A$ & implies & $\tilde{\alpha}_{t} b(A)=b\left(U_{t} A\right)$ \\
\hline$\omega$ is $\left(\alpha_{t}, \beta\right) \mathrm{KMS}$ & implies & $\tilde{\omega}$ is $\left(\tilde{\alpha}_{t}, \beta\right) \mathrm{KMS}$. \\
\hline
\end{tabular}
macro-system resulting from the central limit theorem:

Finally we remark that due to the time invariance of the state: $\omega \circ \alpha_{t}=\omega$, the dynamics $\alpha_{t}$ is implemented in the GNS-representation by

$$
\alpha_{t}(A)=e^{i t H} A e^{-i t H} ; \quad A \in \mathscr{B},
$$

where $H$ is called the Hamiltonian. Denote $U_{t}=e^{i t H}$. Also on the macro level: $\tilde{\omega} \circ \tilde{\alpha}_{t}=\tilde{\omega}$ therefore

$$
\tilde{\alpha}_{t}(W(A))=e^{i t \tilde{H}} W(A) e^{-i t \tilde{H}} .
$$

$\tilde{H}$ is the Hamiltonian of the time evolution on the level of the fluctuations. Denote also $\tilde{U}_{t}=e^{i t \tilde{H}}$.

If $P \in \mathscr{A}_{L, s a}$ one denotes the time evolution perturbed by $P$ as follows:

$$
\alpha_{t}^{P}(A)=e^{i t(H+P)} A e^{-i t(H+P)}, \quad A \in \mathscr{B} .
$$

In the next section we aim at the study of the limit

$$
\lim _{n} \alpha_{t}^{\tilde{P}_{n}}
$$

as a bona fide dynamics of the fluctuation algebra $\tilde{\mathscr{M}}$. 


\section{Perturbations of the Dynamics}

The linear response theory consists in perturbing the dynamics by adding a perturbation to the Hamiltonian and in studying the effect of this perturbation on the expectation values.

On the microscopic level $\left(\mathscr{B}, \omega, \alpha_{t}\right)$, the dynamics perturbed by a bounded perturbation $P \in \mathscr{B}$ is given by the norm convergent Dyson sequence:

$$
\begin{aligned}
\alpha_{t}^{P}(A)= & \alpha_{t}(A)+\sum_{i=1}^{n} i^{n} \int_{0}^{t} d s_{1} \int_{0}^{s_{1}} d s_{2} \cdots \int_{0}^{s_{n}} d s_{n} \\
& \cdot\left[\alpha_{s_{n}}(P), \ldots,\left[\alpha_{s_{1}}(P), \alpha_{t}(A)\right] \ldots\right] .
\end{aligned}
$$

In this section we construct the correspondent perturbed dynamics on the macro-system $\tilde{\mathscr{M}}$. In particular we have to consider the limit

$$
\lim _{n \rightarrow \infty} \tilde{\alpha}_{t}^{\tilde{n}_{n}}\left(\tilde{A}_{n}\right) \text {, }
$$

where $\tilde{P}_{n}$ and $\tilde{A}_{n}$ are the local fluctuations of $P$ and $A$.

By (2.23) for each finite volume the dynamics $\alpha_{t}^{\tilde{P}_{n}}$ corresponds to the Hamiltonian $H+\tilde{P}_{n}$. In this section we prove that the limit dynamics on the fluctuations corresponds to the Hamiltonian

$$
\tilde{H}+b(P),
$$

where $\tilde{H}$ is as in (2.24) and $b(P)$ the macro-fluctuation of $P$ which is linear in the perturbation $P$, i.e. macroscopically the Hamiltonian is already linear in the perturbation. Furthermore we prove that the Dyson expansion (3.1) of the dynamics (3.2) vanishes after the linear term.

Our main tool to prove all this is the following Lemma which is a non-trivial extension of Proposition 6.2.9 of [10].

Lemma 3.1. Suppose that the potential $\phi$ has a finite range $\Lambda_{0}(\phi(X)=0$ if $0 \in X$ and $X \not \Lambda_{0}$ ). Take $B, C \in \mathscr{B}$, then for each finite volume $\tilde{\Lambda}_{0}$ :

$$
\begin{aligned}
\sup _{A \in \mathscr{\alpha} \tilde{\lambda}_{0}} & \frac{\left\|\left[C,\left[\tau_{x} \alpha_{t} A, B\right]\right]\right\|}{\|A\|} \\
\leqq & \sum_{y \in \mathbb{Z}^{v}}\left\{\left(\sum_{z \in \mathbb{Z}^{v}} \sup _{A} \frac{\left\|\left[\tau_{z} A, B\right]\right\|}{\|A\|} e^{-|y-z|}\right)\left(\sum_{z^{\prime} \in \mathbb{Z}^{v}} \sup _{A} \frac{\left\|\left[\tau_{z^{\prime}} A, C\right]\right\|}{\|A\|} e^{-\left|y-z^{\prime}\right|}\right)\right. \\
& \left.+\sup _{A} \frac{\left\|\left[C,\left[\tau_{y} A, B\right]\right]\right\|}{\|A\|}\right\} e^{-|x-y|} e^{|t| K\left(\Lambda_{0}, \tilde{\lambda}_{0}\right)}
\end{aligned}
$$

where $K$ is a finite constant.

Proof. Denote for $A \in \mathscr{A}_{\tilde{\Lambda}_{0}}, B, C \in \mathscr{B}$ :

$$
\begin{aligned}
\xi_{A, B}^{A}(x, t) & =\left[\tau_{x} \alpha_{t}^{\Lambda}(A), B\right], \\
\xi_{B}(x, t) & =\sup _{\boldsymbol{A}} \sup _{A \in \mathscr{A ^ { \prime }} \tilde{\tau}_{0}} \frac{\left\|\xi_{\boldsymbol{A}, \boldsymbol{B}}^{\Lambda}(x, t)\right\|}{\|A\|},
\end{aligned}
$$




$$
\begin{aligned}
\xi_{A, B, C}^{\Lambda}(x, t) & =\left[C,\left[\tau_{x} \alpha_{t}^{\Lambda}(A), B\right]\right], \\
\xi_{B, C}(x, t) & =\sup _{\Lambda} \sup _{A \in \mathscr{A} \tilde{\Lambda}_{0}} \frac{\left\|\xi_{A, B, C}^{\Lambda}(x, t)\right\|}{\|A\|} .
\end{aligned}
$$

Then

$$
\begin{aligned}
\xi_{A, B, C}^{A}(x, t) & =\xi_{A, B, C}^{A}(x, 0)+\int_{0}^{t} d s \frac{d}{d s} \xi_{A, B, C}^{A}(x, s) \\
& =\xi_{A, B, C}^{A}(x, 0)+i \sum_{X \cap \tilde{\Lambda}_{0} \neq \emptyset} \int_{0}^{t} d s\left[C,\left[\tau_{x} \alpha_{s}^{A}([\phi(X), A]), B\right]\right]
\end{aligned}
$$

and

$$
\xi_{B, C}(x, t) \leqq \xi_{B, C}(x, 0)+\sum_{X \cap \tilde{\Lambda}_{0} \neq \emptyset} \int_{0}^{|t|} d s \sup _{\Lambda} \sum_{A \in \mathscr{A}_{\tilde{\Lambda}_{0}}} \frac{\left\|\left[C,\left[\tau_{x} \alpha_{s}^{A}([\phi(X), A]), B\right]\right]\right\|}{\|A\|} .
$$

As in the proof of [10:6.2.9], let $e\left(i_{x}, j_{x}\right)$ be a set of matrix units of $\mathscr{A}_{x}$, then

$$
[\phi(X), A]=\sum_{\left\{i_{x}, j_{x}\right\}} C_{\phi}\left(i_{x}, j_{x}\right) \prod_{x \in X \cup \tilde{\Lambda}_{0}} e\left(i_{x}, j_{x}\right),
$$

where the coefficients $C_{\phi} \in \mathbb{C}$ satisfy

$$
\left|C_{\phi}\left(i_{x}, j_{x}\right)\right| \leqq 2\|\phi(X)\|\|A\| .
$$

After substitution of this decomposition,

$$
\begin{aligned}
& \|\left[C,\left[\tau_{x} \alpha_{s}^{\Lambda}[\phi(X), A], B\right] \|\right. \\
& \quad \leqq 2\|\phi(X)\|\|A\| \sum_{\left\{i_{y}, j_{y}\right\}}\left\|\left[C,\left[\prod_{y \in X \cup \tilde{\Lambda}_{0}} \alpha_{s}^{\Lambda}\left(e\left(i_{x+y}, j_{x+y}\right)\right), B\right]\right]\right\| .
\end{aligned}
$$

After working out the commutators with the product $\prod_{y \in X \cup \Lambda_{0}} \cdots$ one gets

$$
\begin{aligned}
& \|\left[C,\left[\tau_{x} \alpha_{s}^{\Lambda}[\phi(X), A], B\right] \|\right. \\
& \quad \leqq 2\|A\|\|\phi(X)\| \sum_{\left\{i_{y}, j_{y}\right\}}\left\{\sum_{\substack{y, y^{\prime} \in X \cup \tilde{A}_{0} \\
y \neq y^{\prime}}} \xi_{C}(x+y, s) \xi_{B}\left(x+y^{\prime}, s\right)+\sum_{y} \xi_{B, C}(x+y, s)\right\} .
\end{aligned}
$$

Using $\sum_{\left\{i_{y}\right\}} \leqq N^{|X|+\left|\tilde{\Lambda}_{0}\right|}$ and taking the sup over all $A \in \mathscr{A}_{\tilde{\Lambda}_{0}}$ :

$$
\begin{aligned}
\xi_{B, C}(x, t) \leqq & \xi_{B, C}(x, 0)+\sum_{\substack{X \\
X \cap \tilde{\Lambda}_{0} \neq \emptyset}} 2\|\phi(X)\| N^{2\left(|X|+\left|\tilde{\Lambda}_{0}\right|\right)} \\
& \cdot\left\{\sum_{\substack{y, y^{\prime} \in X \cup \tilde{\Lambda}_{0} \\
y \neq y^{\prime}}} \int_{0}^{|t|} d s \xi_{C}(x+y, s) \xi_{B}\left(x+y^{\prime}, s\right)+\sum_{y \in X \cup \tilde{\Lambda}_{0}} \int_{0}^{|t|} d s \xi_{B, C}(x+y, s)\right\} .
\end{aligned}
$$

Using [10] Proposition 6.2.9 in the form:

$$
\xi_{B}(x, t) \leqq \sum_{y \in \mathbb{Z}^{v}} \xi_{B}(y, 0) e^{-|x-y|} e^{|t| \tilde{K}\left(\Lambda_{0}, \tilde{\Lambda}_{0}\right)}
$$


yields

$$
\begin{aligned}
\sum_{\substack{y, y^{\prime} \in X \cup y^{\prime} \\
y \neq y^{\prime}}} \int_{0}^{|t|} \xi_{C}(x+y, s) \xi_{B}\left(x+y^{\prime}, s\right) \leqq & \sum_{\substack{y, y^{\prime} \in X \cup y^{\prime} \\
y \neq y^{\prime}}} \sum_{\substack{\lambda^{\prime} \\
z, z^{\prime} \in \mathbb{Z}^{v}}} \int_{0}^{|t|} d s \xi_{C}(z, 0) \xi_{B}\left(z^{\prime}, 0\right) \\
& \cdot \exp \left\{-|x+y-z|-\left|x+y^{\prime}-z^{\prime}\right|+2 \tilde{K}\left(\Lambda_{0}, \tilde{\Lambda}_{0}\right) s\right\} .
\end{aligned}
$$

As

$$
|x-z|-|y| \leqq|x-z+y|
$$

the first group of terms of $(*)$ is bounded by

$$
\begin{gathered}
\sum_{\substack{X \\
x \cap \tilde{\Lambda}_{0} \neq \varnothing}} 2\|\phi(X)\| N^{2\left(|X|+\left|\tilde{\Lambda}_{0}\right|\right)} \sum_{\substack{y, y^{\prime} \in X \cup \tilde{A}_{0} \\
y \neq \tilde{y}^{\prime}}} e^{|y|+\left|y^{\prime}\right|} \\
\sum_{z, z^{\prime}} \xi_{C}(z, 0) \xi_{B}\left(z^{\prime}, 0\right) e^{-|x-z|-\left|x-z^{\prime}\right|} \int_{0}^{|t|} d s e^{2 s \tilde{K}\left(\Lambda_{0}, \tilde{A}_{0}\right)} .
\end{gathered}
$$

Recall that the interaction is of finite range $\Lambda_{0}$ and that $\tilde{\Lambda}_{0}$ is finite. This yields that the sums $\sum_{X}$ and $\sum_{y, y^{\prime} \in X \cup \tilde{\Lambda}_{0}}$ are finite, adding up to a constant $M\left(\Lambda_{0}, \tilde{\Lambda}_{0}\right)$ such that the first group of terms of $(*)$ is bounded by

$$
\begin{aligned}
\leqq & M\left(\Lambda_{0}, \tilde{\Lambda}_{0}\right) \sum_{z, z^{\prime} \in \mathbb{Z}^{v}} \xi_{C}(z, 0) \xi_{B}\left(z^{\prime}, 0\right) e^{-|x-z|-\left|x-z^{\prime}\right|} \\
& \cdot \frac{e^{2|t| \tilde{K}\left(\Lambda_{0}, \tilde{\Lambda}_{0}\right)}-1}{2 \widetilde{K}\left(\Lambda_{0}, \tilde{\Lambda}_{0}\right)} .
\end{aligned}
$$

About the second group of terms in $(*)$, it is clear that there exists a constant $M^{\prime}\left(\Lambda_{0}, \tilde{\Lambda}_{0}\right)$ and a finite volume $\Gamma_{0}$ such that one has the bound

$$
M^{\prime}\left(\Lambda_{0}, \tilde{\Lambda}_{0}\right) \sum_{y \in \Gamma_{0}} \int_{0}^{|t|} d s \xi_{B, c}(x+y, s)
$$

Denoting

$$
\begin{aligned}
\mathscr{G}_{B, C}(x) & =M \sum_{z, z^{\prime}} \xi_{C}(z, 0) \xi_{B}\left(z^{\prime}, 0\right) e^{-|x-z|-\left|x-z^{\prime}\right|} \\
F(t) & =\frac{e^{2|t| \tilde{K}}-1}{2 \tilde{K}}
\end{aligned}
$$

then

$$
\xi_{B, C}(x, t) \leqq \xi_{B, C}(x, 0)+\mathscr{G}_{B, C}(x) F(t)+M^{\prime} \sum_{y \in \Gamma_{0}} \int_{0}^{|t|} d s \xi_{B, C}(x+y, s) .
$$

After iteration

$$
\begin{aligned}
\xi_{B, C}(x, t) \leqq & \sum_{n=0}^{\infty} \frac{M^{\prime n}|t|^{n}}{n !} \sum_{y_{1}, \ldots, y_{n} \in \Gamma_{0}} \xi_{B, C}\left(x+y_{1}+\cdots+y_{n}, 0\right) \\
& +\sum_{n=0}^{\infty} M^{\prime n}\left(\sum_{y_{1}, \ldots, y_{n} \in \Gamma_{0}} \mathscr{G}_{B, C}\left(x+y_{1}+\cdots+y_{n}\right)\right)\left(\int_{0}^{|t|} d s_{1} \cdots \int_{0}^{s_{n}-1} d s_{n} F\left(s_{n}\right)\right) .
\end{aligned}
$$


Insert the bound

$$
\int_{0}^{|t|} d s_{1} \cdots \int d s_{n} F\left(s_{n}\right) \leqq|t| \frac{e^{\tilde{K} t|t|^{n}}}{n !}
$$

and use the Fourier representation technique of the proof of [10] Proposition 6.2.9 to finish the proof of this Lemma.

Corollary 3.2. If $A, B, C \in \mathscr{A}_{\tilde{\Lambda}_{0}}$, then there exists a constant $L\left(\Lambda_{0}, \tilde{\Lambda}_{0}\right)$ such that

$$
\sum_{x, y \in \mathbb{Z}^{v}}\left\|\left[\alpha_{t} \tau_{x} A,\left[\alpha_{s} \tau_{y} B, C\right]\right]\right\| \leqq L\left(\Lambda_{0}, \tilde{\Lambda}_{0}\right) e^{2(|t|+|s|) K} .
$$

Proof. Use $[10 ; 6.2 .9]$ in the form

$$
\sup _{A \in \mathscr{A} \tilde{\lambda}_{0}} \frac{\left\|\left[\tau_{x} \alpha_{t} A, B\right]\right\|}{\|A\|} \leqq \sum_{y \in \mathbb{Z}^{v}} \sup _{A \in \mathscr{A} \tilde{\lambda}_{0}} \frac{\left\|\left[\tau_{y} A, B\right]\right\|}{\|A\|} e^{-|x-y|} e^{|t| K}
$$

for $B \in \mathscr{B}$ to obtain by replacing $B$ by $\left[\alpha_{s} \tau_{y} B, C\right]$

$$
\left\|\left[\alpha_{t} \tau_{x} A,\left[\alpha_{s} \tau_{y} B, C\right]\right]\right\| \leqq\|A\| \sum_{z \in \mathbb{Z}^{v}} \sup _{D \in \mathscr{A} \tilde{\lambda}_{0}} \frac{\left\|\left[\tau_{z} D,\left[\alpha_{s} \tau_{y} B, C\right]\right]\right\|}{\|D\|} e^{-|x-z|} e^{|t| K} .
$$

Using Lemma 3.1 and after performing the summation $\sum_{x, y}$ one gets the bound

$$
\begin{aligned}
& \|A\|\|B\|\left(\sum_{x} e^{-|x|}\right)^{2} \sum_{z, z^{\prime}} \sup _{D, E \in \mathscr{A} \tilde{\lambda}_{0}} \frac{\left\|\left[\tau_{z} D,\left[\tau_{z^{\prime}} E, C\right]\right]\right\|}{\|D\|\|E\|} e^{(|t|+|s| K} \\
& \quad+\|A\|\|B\|\left(\sum_{x} e^{-|x|}\right)^{4}\left(\sum_{z} \sup _{D, E} \frac{\left\|\left[E, \tau_{z} D\right]\right\|}{\|E\|\|D\|}\right)\left(\sum_{z} \sup _{E} \frac{\left\|\left[\tau_{z} E, C\right]\right\|}{\|E\|}\right) e^{2(|t|+|s|) K},
\end{aligned}
$$

proving the corollary.

Now one is able to derive a bound for the fluctuations.

Corollary 3.3. If $A, B, C \in \mathscr{A}_{\tilde{\Lambda}_{0}}$ and

$$
\tilde{A}_{n}=\frac{1}{\left|\Lambda_{n}\right|^{1 / 2}} \sum_{x \in \Lambda_{n}}\left(\tau_{x} A-\omega(A)\right), \quad \tilde{B}_{n}=\cdots
$$

then for all $t, s \in \mathbb{R}$ :

$$
\left\|\left[\alpha_{t} \tilde{A}_{n},\left[\alpha_{s} \tilde{B}_{n}, \tilde{C}_{n}\right]\right]\right\| \leqq 0\left(\frac{1}{\left|\Lambda_{n}\right|^{1 / 2}}\right)
$$

Proof.

$$
\begin{aligned}
\left\|\left[\alpha_{t} \tilde{A}_{n},\left[\alpha_{s} \tilde{B}_{n}, \tilde{C}_{n}\right]\right]\right\| & \leqq \frac{1}{\left|\Lambda_{n}\right|^{3 / 2}} \sum_{x, y, z \in \Lambda_{n}}\left\|\left[\alpha_{t} \tau_{x} A,\left[\alpha_{x} \tau_{y} B, \tau_{z} C\right]\right]\right\| \\
& \leqq \frac{1}{\left|\Lambda_{n}\right|^{1 / 2}} \sum_{x, y \in \Lambda_{2 n}}\left\|\left[\alpha_{t} \tau_{x} A,\left[\alpha_{s} \tau_{y} B, C\right]\right]\right\| \\
& \leqq \frac{L}{\left|\Lambda_{n}\right|^{1 / 2}} e^{2(|t|+|s|) K}
\end{aligned}
$$

where we used 3.2 in the last step. 
Using this bound on the commutators of fluctuations one can prove the following central limit theorem yielding the existence and explicit form of the perturbed dynamics on the macroscopic fluctuations.

Theorem 3.4 (central limit theorem). If the microsystem $\left(\mathscr{B}, \omega, \alpha_{t}\right)$ satisfies the following conditions:

(i) the dynamics $\alpha_{t}$ is of finite range,

(ii) the state $\omega$ is time- and space-translation invariant,

(iii) the state $\omega$ satisfies the cluster condition (2.8),

then, using the notations of Sect. 2, for all $A, B, P \in \mathscr{A}_{L, s a}$ :

$$
\lim _{n} \omega\left(e^{i \tilde{A}_{n}} \alpha_{t}^{\tilde{P}_{n}}\left(e^{i \tilde{B}_{n}}\right) e^{-i \tilde{A}_{n}}\right)=\tilde{\omega}\left(W(A) \tilde{\alpha}_{t}^{P}(W(B)) W(-A)\right)
$$

where $\left\{\tilde{\alpha}_{t}^{P}\right\}_{t}$ is a one-parameter group of *-automorphisms of $\tilde{\mathscr{M}}$, explicitly given by

$$
\tilde{\alpha}_{t}^{P}(\cdot)=W\left(P^{t}\right) \tilde{\alpha}_{t}(\cdot) W\left(-P^{t}\right)
$$

where $P^{t}=\int_{0}^{t} d s U_{s} P$.

Proof. If $X^{*}=X, Y^{*}=Y$ then one has the bounds:

$$
\begin{aligned}
& \left\|e^{i(X+Y)}-e^{i X}\right\| \leqq\|Y\|, \\
& \left\|e^{i(X+Y)}-e^{i X} e^{i Y}\right\| \leqq \frac{1}{2}\|[X, Y]\|, \\
& \left\|\left[e^{i X}, e^{i Y}\right]\right\| \leqq\|[X, Y]\| .
\end{aligned}
$$

Iterating the Dyson integral formula:

$$
\begin{aligned}
\alpha_{t}^{\tilde{P}_{n}}\left(\tilde{A}_{n}\right) & =X_{n}+Y_{n} \\
X_{n} & =\alpha_{t}\left(\tilde{A}_{n}\right)+i \int_{0}^{t} d s\left[\alpha_{s}\left(\tilde{P}_{n}\right), \alpha_{t}\left(\tilde{A}_{n}\right)\right], \\
Y_{n} & =i^{2} \int_{0}^{t} d s_{1} \int_{0}^{s_{1}} d s_{2} \alpha_{s}^{\tilde{P}_{n}} \alpha_{-s_{2}}\left[\alpha_{s_{2}}\left(\tilde{P}_{n}\right),\left[\alpha_{s_{1}}\left(\tilde{P}_{n}\right), \alpha_{t}\left(\tilde{A}_{n}\right)\right]\right],
\end{aligned}
$$

and for $A^{*}=A$ :

$$
\begin{aligned}
\left\|\alpha_{t}^{\tilde{P}_{n}}\left(e^{i \tilde{A}_{n}}\right)-e^{i X_{n}}\right\| & =\left\|e^{i \alpha_{n} \tilde{P}_{n}\left(\tilde{A}_{n}\right)}-e^{i X_{n}}\right\| \\
& =\left\|e^{i\left(X_{n}+Y_{n}\right)}-e^{i X_{n}}\right\| \leqq\left\|Y_{n}\right\| \\
& \leqq \int_{0}^{|t|} d s_{1} \int_{|t|}^{|t|} d s_{1}\left\|\left[\alpha_{s_{2}}\left(\tilde{P}_{n}\right),\left[\alpha_{s_{1}}\left(\tilde{P}_{n}\right), \tilde{A}_{n}\right]\right]\right\| \\
& \leqq O\left(\frac{1}{\left|\Lambda_{n}\right|^{1 / 2}}\right)
\end{aligned}
$$

where Corollary 3.3 is used for the last inequality. This means that

$$
\lim _{n}\left\|\alpha_{t}^{\tilde{P}_{n}}\left(e^{i \tilde{A}_{n}}\right)-e^{i X_{n}}\right\|=0
$$

or, if the limits exist:

$$
\lim _{n} \alpha_{t}^{\tilde{P}_{n}}\left(e^{i \tilde{A}_{n}}\right)=\lim _{n} e^{i X_{n}}
$$


For $X=X^{*} \in \mathscr{B}$, denote

$$
\tau_{t X}(Y)=e^{i t X} Y e^{-i t X}
$$

Clearly one has

$$
\begin{aligned}
\tau_{t X}(Y) & =Y+i \int_{0}^{t} d s \tau_{s X}([X, Y]) \\
& =Y+i t[X, Y]+i^{2} \int_{0}^{t} d s \int_{0}^{s} d s^{\prime} \tau_{s^{\prime} X}([X,[X, Y]])
\end{aligned}
$$

Now we turn to the proof of the existence of the central limit. Denote

$$
I=\lim _{n \rightarrow \infty} \omega\left(e^{i \tilde{A}_{n}} \alpha_{t}^{\tilde{P}_{n}}\left(e^{i \tilde{B}_{n}}\right) e^{-i \tilde{A}_{n}}\right) .
$$

Using Corollary 3.3 and respectively (d) and (b),

$$
\begin{aligned}
I & =\lim _{n \rightarrow \infty} \omega\left(e^{i \tilde{A}_{n}} e^{l\left(\alpha_{t}\left(\tilde{B}_{n}\right)+i\left[\tilde{P}_{n}^{t}, \alpha_{t} \tilde{B}_{n}\right]\right)} e^{-i \tilde{A}_{n}}\right) \\
& =\lim _{n \rightarrow \infty} \omega(\underbrace{\underbrace{i \tilde{A}_{n}} e^{i \alpha_{t} \tilde{B}_{n}} e^{-i \tilde{A}_{n}} e^{i \tilde{A}_{n}} \underbrace{e^{2}\left[\tilde{P}_{n}^{t}, \alpha_{t} \tilde{B}_{n}\right]} e^{-i \tilde{A}_{n}}}),
\end{aligned}
$$

where $\tilde{P}_{n}^{t}=\int_{0}^{t} d s \alpha_{s} \widetilde{P}_{n}$.

Because $\tau_{t X}\left(e^{i Y}\right)=e^{i \tau_{t X}(Y)}$ and using (a), Corollary (3.3) and (f) for the first factor and (e) for the second one, we obtain

$$
\begin{aligned}
I & =\lim _{n \rightarrow \infty} \omega\left(e^{i\left(\alpha_{t} \tilde{B}_{n}+i\left[\tilde{A}_{n}, \alpha_{t} \tilde{B}_{n}\right]\right)} e^{i^{2}\left[\tilde{P}_{n}^{t}, \alpha_{t} \tilde{B}_{n}\right]}\right) \\
& =\lim _{n \rightarrow \infty} \omega\left(\alpha_{t}\left(e^{i \tilde{B}_{n}}\right) e^{-\left[i \tilde{A}_{n}, \alpha_{t} \tilde{B}_{n}\right]} e^{-\left[\tilde{P}_{n}^{t}, \alpha_{t} \tilde{B}_{n}\right]}\right) .
\end{aligned}
$$

In the last step we used again (b) and the corollary. Remains to prove this limit $n \rightarrow \infty$. As an immediate consequence of [10] Proposition 6.2.9

$$
\sum_{x \in \mathbb{Z}^{v}}\left\|\left[\tau_{x} \alpha_{t} A, B\right]\right\|<\infty
$$

yielding

$$
\sum_{x}\left[\tau_{x} \alpha_{t} A, B\right] \in \mathscr{B} .
$$

Because of the clusterproperty (2.8), the state $\omega$ is ergodic implying that for all $C \in \mathscr{B}$, the following weak operator limit in the GNS-representation induced by $\omega$, yields:

$$
\text { weak- } \lim _{\Lambda} C_{\Lambda}=\omega(C)
$$

where $C_{\Lambda}=\frac{1}{|\Lambda|} \sum_{x \in \Lambda} \tau_{x} C$, and straightforwardly

$$
\lim _{\Lambda} \omega\left(X e^{C_{\Lambda}}\right)=e^{\omega(C)} \omega(X) \text { for all } X \in \mathscr{B} .
$$

In particular this argument gives

$$
I=\tilde{\omega}(W(B)) \exp \left\{-i \sigma_{\omega}\left(A, \alpha_{t} B\right)-i \sigma_{\omega}\left(P^{t}, B\right)\right\}
$$


A simple CCR-computation and (5), (7) yield the formulae

$$
\lim _{n} \omega\left(e^{i \tilde{A}_{n}} \alpha_{t}^{\tilde{P}_{n}}(W(B)) e^{-i \tilde{A}_{n}}\right)=\tilde{\omega}\left(W(A) \tilde{\alpha}_{t}^{P}(W(B)) W(-A)\right),
$$

where

$$
\tilde{\alpha}_{t}^{P}(\cdot)=W\left(P^{t}\right) \tilde{\alpha}_{t}(\cdot) W\left(-P^{t}\right)
$$

Clearly $\left\{\tilde{\alpha}_{t}^{P}\right\}_{t}$ is a one-parameter family of $*$-automorphisms of $\tilde{\mathscr{M}}$. Finally we check the group property:

$$
\tilde{\alpha}_{t_{1}}^{P} \tilde{\alpha}_{t_{2}}^{P}(X)=W\left(P^{t_{1}}\right) \tilde{\alpha}_{t_{1}}\left(W\left(P^{t_{2}}\right)\right) \tilde{\alpha}_{t_{1}+t_{2}}(X) \tilde{\alpha}_{t_{1}}\left(W\left(-P^{t_{2}}\right)\right) W\left(-P^{t_{1}}\right) .
$$

But

$$
\begin{aligned}
W\left(P^{t_{1}}\right) \tilde{\alpha}_{t_{1}} W\left(P^{t_{2}}\right) & =W\left(P^{t_{1}}\right) W\left(\int_{t_{1}}^{t_{2}+t_{1}} d s U_{s} P\right) \\
& =W\left(P^{t_{1}+t_{2}}\right) \exp -\frac{i}{2} \sigma_{\omega}\left(P^{t_{1}}, P^{t_{1}+t_{2}}\right)
\end{aligned}
$$

Hence

$$
\tilde{\alpha}_{t_{1}}^{P} \tilde{\alpha}_{t_{2}}^{P}=\tilde{\alpha}_{t_{1}+t_{2}}^{P}
$$

proving the theorem.

We study somewhat more in detail the properties of the perturbed macrodynamics $\tilde{\alpha}_{t}^{P}$ obtained in the preceding theorem.

Under the same condition of Theorem 3.4 we prove

Theorem 3.5. For all $t \in \mathbb{R}, \tilde{\alpha}_{t}^{P}$ is implemented by the unitary operators

$$
\tilde{U}_{t}^{P}=\exp i t(\tilde{H}+b(P))
$$

i.e. $\tilde{\alpha}_{t}^{P}(\cdot)=\tilde{U}_{t}^{P} \cdot \tilde{U}_{-t}^{P}$

Proof. From 3.4 and (2.24) one gets

$$
\tilde{\alpha}_{t}^{P}(\cdot)=\tilde{U}_{t}^{P} \cdot \tilde{U}_{-t}^{P}
$$

where

$$
\tilde{U}_{t}^{P}=\exp \left\{-\frac{i}{2} \int_{0}^{t} \sigma_{\omega}\left(P, P^{s}\right) d s\right\} W\left(P^{t}\right) e^{i t \tilde{H}} .
$$

We prove that $\left\{\tilde{U}_{t}^{P}\right\}_{t \in \mathbb{R}}$ is a strongly continuous one-parameter group of unitaries on $\tilde{\mathscr{H}}$. First the group property: using the CCR-calculus,

$$
\begin{aligned}
\tilde{U}_{t}^{P} \tilde{U}_{t^{\prime}}^{P}= & W\left(P^{t}+\alpha_{t} P^{t^{\prime}}\right) e^{i\left(t+t^{\prime}\right) \tilde{H}} \\
& \cdot \exp -\frac{i}{2}\left\{\sum_{0}^{t} d s \sigma_{\omega}\left(P, P^{s}\right)+\int_{0}^{t} d s \sigma_{\omega}\left(P, P^{s}\right)+\sigma_{\omega}\left(P^{t}, \alpha_{t} P^{t^{\prime}}\right)\right\} .
\end{aligned}
$$

Furthermore

$$
P^{t}+\alpha_{t} P^{t^{\prime}}=P^{t+t^{\prime}}
$$


and $\sigma_{\omega}\left(P_{t}, \alpha_{t} P_{t}^{\prime}\right)=\int_{0}^{t^{\prime}} d s\left(\sigma_{\omega}\left(P, P^{t+s}\right)-\sigma_{\omega}\left(P, P^{s}\right)\right)$, yielding

$$
\left(\int_{0}^{t}+\int_{0}^{t^{\prime}}\right) d s \sigma_{\omega}\left(P, P^{s}\right)+\sigma_{\omega}\left(P^{t}, \alpha_{t} P^{t^{\prime}}\right)=\int_{0}^{t+t^{\prime}} d s \sigma_{\omega}\left(P, P^{s}\right) .
$$

Hence

$$
\tilde{U}_{t}^{P} \tilde{U}_{t^{\prime}}^{P}=\tilde{U}_{t+t^{\prime}}^{P}
$$

As $\tilde{\omega}$ is a quasi-free state, the strong continuity of

$$
t \rightarrow \tilde{U}_{t}^{P}
$$

is immediate.

Finally, using (2.17) one gets

$$
\left.\frac{d}{i d t} \tilde{U}_{t}^{P}\right|_{t=0}=\tilde{H}+b(P) .
$$

From above we have that

$$
\tilde{\alpha}_{t}^{P}(\cdot)=\tilde{U}_{t}^{P} \cdot \tilde{U}_{-t}^{P}=\Gamma_{t}^{P} \tilde{\alpha}_{t}(\cdot)\left(\Gamma_{t}^{P}\right)^{*}
$$

where

$$
\Gamma_{t}^{P}=e^{i t(\tilde{H}+b(P))} e^{-i t \tilde{H}}=\exp \left\{-\frac{i}{2} \int_{0}^{t} d s \sigma_{\omega}\left(P, P^{s}\right)\right\} W\left(P^{t}\right)
$$

Remark that

$$
\Gamma_{t}^{P} \in \tilde{\mathscr{M}}, \quad t \in \mathbb{R}
$$

and satisfies the cocycle relation:

$$
\Gamma_{t+s}^{P}=\Gamma_{t}^{P} \tilde{\alpha}_{t}\left(\Gamma_{s}^{P}\right) ; \quad s, t \in \mathbb{R} .
$$

This finishes the proof of the existence of the perturbed dynamics on the macroscopic algebra of fluctuations and its mathematical properties.

In physics [3-8] one considers the evolution of an expectation value under the perturbed evolution, i.e. in our notation one considers the expression

$$
\omega\left(\alpha_{t}^{P}(A)\right)-\omega(A)
$$

for $A, P \in \mathscr{A}_{L, s a}$ as a function of $t$. This function is called the response function. Using formula (3.1) this function is a nonlinear function of $P$. Linear response theory consists in approximating the series (3.1) by limiting it to the linear term in the perturbation observable $P$.

The mathematics above learns now the following. If one considers a fluctuation

$$
\tilde{P}_{n}=\frac{1}{\left|\Lambda_{n}\right|^{1 / 2}} \sum_{x \in \Lambda_{n}}\left(\tau_{x} P-\omega(P)\right)
$$

instead of a local perturbation $P \in \mathscr{A}_{L, s a}$ and if one computes the time evolution of a fluctuation $\tilde{A}_{n}$ instead of a local observable $A \in \mathscr{A}_{L, s a}$ i.e. instead we consider the 
response function

$$
\omega\left(\alpha_{t}^{\tilde{P}_{n}}\left(\tilde{A}_{n}\right)\right)
$$

then one has:

$$
\begin{aligned}
\lim _{n \rightarrow \infty} \omega\left(\tilde{\alpha}_{t}^{\tilde{P}_{n}}\left(\tilde{A}_{n}\right)\right) & =-\int_{0}^{t} d s \sigma_{\omega}\left(P, U_{s} A\right)=\tilde{\omega}\left(\tilde{\alpha}_{t}^{P}(b(A))\right) \\
& =i \int_{0}^{t} d s \tilde{\omega}\left(\left[b(P), \tilde{\alpha}_{s} b(A)\right]\right) .
\end{aligned}
$$

These formulae are a simple consequence of Theorem 3.4.

One reads off from these formulae that in our case the total response equals the linear response or in other words we proved that the linear response theory becomes exact for all values of the time parameter.

Remark that this result holds for all stationary states. The state $\omega$ need not be an equilibrium state. Of course in the latter case one has more structure, and this will be the subject of the next section.

\section{Linear Response for Equilibrium States}

In [12] we proved the following property. If the microsystem is in an equilibrium state, i.e. the state $\omega$ is an $\left(\alpha_{t}, \beta\right)-$ KMS state of $\mathscr{B}$, then also the macrostate $\tilde{\omega}$ of the fluctuation algebra $\tilde{\mathscr{M}}$ is in an equilibrium state at the same temperature, i.e. the state $\tilde{\omega}$ is an $\left(\tilde{\alpha}_{t}, \beta\right)-$ KMS state of $\tilde{\mathscr{M}}$. In other words the notion of equilibrium is preserved under the operation of coarse graining determined by the central limit.

Therefore in this section we assume that $\omega$ is an $\left(\alpha_{t}, \beta\right)$ - KMS state of $\mathscr{B}$.

Above we constructed a new dynamics $\tilde{\alpha}_{t}^{P}$ on $\tilde{\mathscr{M}}$ the so-called perturbed dynamics, which on the basis of Sect. 3, can be called the linear response dynamics.

Here we construct the $\left(\tilde{\alpha}_{t}^{P}, \beta\right)$ - KMS state or the equilibrium state for the perturbed dynamics. This will enable us to describe the explicit connection between perturbing the dynamics and perturbing the equilibrium state.

First we show that the perturbed equilibrium state on $\widetilde{\mathscr{M}}$ is given by

$$
\tilde{\omega}^{P}: W(A) \in \tilde{\mathscr{M}} \rightarrow \tilde{\omega}^{P}(W(A))=\tilde{\omega}(W(A)) e^{-i \beta(b(P), b(A))},
$$

where the $W(A)$ are the Weyl operators generating the algebra $\tilde{\mathscr{M}} \tilde{\omega}$ the $\left(\tilde{\alpha}_{t}, \beta\right)$ - KMS state on $\tilde{\mathscr{M}}$, and $(\cdot, \cdot)$ the Duhamel two-point function. Remark that the latter one is well defined on $\tilde{\mathscr{M}}$ (see [6]), but that in order to give (4.1) a precise mathematical meaning, we have to extend it to the unbounded field operators $b(A)$. It turns out that this extension holds on the basis of $\tilde{\omega}$ being a $\left(\tilde{\alpha}_{t}, \beta\right)$ - KMS state and the Tomita - Takesaki theory [10].

Let $\mathscr{M}$ be a von Neumann algebra with a cyclic and separating vector $\Omega$ and $\mathscr{M}^{\prime}$ the commutant of $\mathscr{M}$. Let $F$ and $S$ be the closed extensions of the maps

$$
\begin{array}{ll}
F_{0}: A^{\prime} \Omega \rightarrow A^{\prime *} \Omega, & A^{\prime} \in \mathscr{M}^{\prime} \\
S_{0}: A \Omega \rightarrow A^{*} \Omega, & A \in \mathscr{M}
\end{array}
$$


and

$$
F^{*}=S=J \Delta^{1 / 2} \text { their polar decomposition. }
$$

$\Delta$ is the modular operator of the pair $(\mathscr{M}, \Omega)$ and $J$ the modular conjugation.

Let the operator $B=B^{*}$ be affiliated to $\mathscr{M}$ i.e. $\mathscr{M}^{\prime} \mathscr{D}(B) \subseteq \mathscr{D}(B)$ and $B A^{\prime} \supseteqq A^{\prime} B$ for all $A^{\prime} \in \mathscr{M}^{\prime}$, and let $\Omega \in \mathscr{D}(B)$, then $B \Omega \in \mathscr{D}\left(\Delta^{1 / 2}\right)=\mathscr{D}(S)$. Indeed, for any $A^{\prime} \in \mathscr{M}^{\prime}$ :

$$
\begin{aligned}
\left(B \Omega, F A^{\prime} \Omega\right)= & \left(B \Omega, A^{\prime *} \Omega\right)=\left(A^{\prime} B \Omega, \Omega\right) \\
& =\left(B A^{\prime} \Omega, \Omega\right)=\left(A^{\prime} \Omega, B \Omega\right)=\overline{\left(B \Omega, A^{\prime} \Omega\right)} \\
& =\overline{\left(F^{*} B \Omega, A^{\prime} \Omega\right)}=\overline{\left(S B \Omega, A^{\prime} \Omega\right)} .
\end{aligned}
$$

As $\tilde{\omega}$ is $\left(\tilde{\alpha}_{t}, \beta\right)-\mathrm{KMS}$, the pair $(\tilde{\mathscr{M}}, \tilde{\Omega})$ satisfies the Tomita-Takesaki theory with modular operator $\tilde{\Delta}=\exp -\beta \tilde{H}$.

For any $A \in \mathscr{K}_{\omega}^{\mathrm{Re}}$, the field operator $b(A)$ is unbounded, but affiliated to $\tilde{\mathscr{M}}$ as it is the infinitesimal generator of the one-parameter group of unitaries $\{W(\lambda A) \mid \lambda \in \mathbb{R}\}$ in $\tilde{\mathscr{M}}$,

$$
W(\lambda A)=\exp i \lambda b(A)
$$

Moreover $b(A)^{*}=b(A)$.

From the analyticity of the map

$$
\begin{aligned}
\lambda \rightarrow \tilde{\omega}(W(\lambda A)) & =\left(\Omega_{s}, e^{i \lambda b(A)} \Omega_{s}\right) \\
& =\exp \left\{-\frac{\lambda^{2}}{2}\langle A, A\rangle_{s}\right\},
\end{aligned}
$$

it follows that $\widetilde{\Omega} \in \mathscr{D}(b(A))$. Hence from the argument above we have

$$
b(A) \tilde{\Omega} \in \mathscr{D}\left(\tilde{\Delta}^{1 / 2}\right) .
$$

As $\mathscr{D}\left(\tilde{\Delta}^{t / 2}\right) \subseteq \mathscr{D}\left(\tilde{\Delta}^{1 / 2}\right)$ for all $t \in[0,1]$, the Duhamel two-point function can be extended to the field operators by the following formula:

$$
\left(b\left(A_{1}\right), b\left(A_{2}\right)\right)_{\sim}=\frac{1}{\beta} \int_{0}^{\beta} d t\left(\tilde{\Delta}^{t / 2} b\left(A_{1}\right) \tilde{\Omega}, \tilde{\Delta}^{t / 2} b\left(A_{2}\right) \tilde{\Omega}\right)
$$

for all $A_{1}, A_{2} \in \mathscr{K}_{\omega}^{\mathrm{Re}}$ and also $\left(b\left(A_{1}\right), b\left(A_{2}\right)\right)_{\sim} \in \mathbb{R}$.

Now we are in a position to prove:

Theorem 4.1. If the conditions of Theorem 3.4 are satisfied and if $\omega$ is an $\left(\alpha_{t}, \beta\right)-\mathrm{KMS}$ state, then the state $\tilde{\omega}^{P}$ on $\tilde{\mathscr{M}}$ defined by

$$
\tilde{\omega}^{P}(W(A))=\tilde{\omega}(W(A)) e^{-i \beta(b(P), b(A))}, \quad A \in \mathscr{K}_{\omega}^{\mathrm{Re}}
$$

is an $\left(\tilde{\alpha}_{t}, \beta\right)$ - KMS state.

Proof. We have to prove that for all $X, Y \in \tilde{\mathscr{M}}$, there exists a complex function $F_{X, Y}$ which is analytic on the strip $D_{\beta}=\{z \in \mathbb{C} \mid O<\operatorname{Im} z<\beta\}$, bounded and continuous on the closure $\bar{D}_{\beta}$ and such that

$$
\begin{aligned}
F_{X, Y}(t) & =\tilde{\omega}^{P}\left(X \tilde{\alpha}_{t}^{P} Y\right), \\
F_{X, Y}(t+i \beta) & =\tilde{\omega}^{P}\left(\tilde{\alpha}_{t}^{P}(Y) X\right)
\end{aligned}
$$

for all $t \in \mathbb{R}$. 
Using a standard density argument (see [10] Proof of Proposition 5.3.7) it is sufficient to prove the above statement for a dense subset of $\tilde{\mathscr{M}}$ generated by the Weyl operators $W(A), A \in \mathscr{K}_{\omega, \alpha}^{\mathrm{Re}}$, where $\mathscr{K}_{\omega, \alpha}^{\mathrm{Re}}$ is the subspace of $\mathscr{K}_{\omega}^{\mathrm{Re}}$ generated by the elements

$$
\begin{aligned}
A\left(f_{n}^{u}\right) & =\int d t f_{n}^{u}(t) U_{t} A ; A \in \mathscr{A}_{L}, n \in \mathbb{N}_{0}, \\
f_{n}^{u}(t) & =f_{n}(t-u), u \in \mathbb{R}, \\
f_{n}(t) & =\sqrt{\frac{n}{\pi}} e^{-n t^{2}} .
\end{aligned}
$$

$\mathscr{K}_{\omega, \alpha}^{\mathrm{Re}}$ are the analytic vectors for the evolution $\left(U_{t}\right)_{t}$ (see [12]).

For any $A_{1}, A_{2} \in \mathscr{K}_{\omega, \alpha}^{\mathrm{Re}}$ consider the function

$$
F_{A_{1}, A_{2}}(t)=\tilde{\omega}^{P}\left(W\left(A_{1}\right) \tilde{\alpha}_{t}^{P} W\left(A_{2}\right)\right)
$$

then

$$
F_{A_{1}, A_{2}}(t)=\tilde{\omega}\left(W\left(A_{1}\right) \tilde{\alpha}_{t} W\left(A_{2}\right)\right) \exp -i \beta\left(b(P), b\left(A_{1}\right)+\tilde{\alpha}_{t} b\left(A_{2}\right)\right)_{\sim}+i \sigma_{\omega}\left(U_{t} A_{2}, P^{t}\right) .
$$

Remark that

$$
\sigma_{\omega}\left(U_{t} A_{2}, P^{t}\right)=\sigma_{\omega}\left(A_{2}^{t}, P\right),
$$

where again $A^{t}=\int_{0}^{t} d s U_{t} A$

As $A_{1}, A_{2} \in \mathscr{K}_{\omega, \alpha}^{\mathrm{Re}}$ the function in the exponential extends analytically to the strip $D_{\beta}$. As $\tilde{\omega}$ is $\left(\tilde{\alpha}_{t}, \beta\right)-$ KMS also the function

$$
t \rightarrow \tilde{\omega}\left(W\left(A_{1}\right) \tilde{\alpha}_{t} W\left(A_{2}\right)\right)
$$

shares this property and in fact this is the full KMS-condition.

Therefore it remains to prove that

$$
F_{A_{1}, A_{2}}(i \beta)=\tilde{\omega}^{P}\left(W\left(A_{2}\right) W\left(A_{1}\right)\right)
$$

or equivalently

$$
-i \beta\left(b(P), \tilde{\alpha}_{i \beta} b\left(A_{2}\right)\right)_{\sim}+i \sigma_{\omega}\left(A_{2}^{t=i \beta}, P\right)=-i \beta\left(b(P), b\left(A_{2}\right)\right)_{\sim} .
$$

Remark that

$$
i \sigma_{\omega}\left(A_{2}^{t}, P\right)=\int_{0}^{t} d s \tilde{\omega}\left(\left[\tilde{\alpha}_{s} b\left(A_{2}\right), b(P)\right]\right) .
$$

Using again the KMS-property of $\tilde{\omega}$ one gets

$$
\begin{aligned}
-i \beta\left(b(P), \tilde{\alpha}_{i \beta} b\left(A_{2}\right)\right)_{\sim}+i \sigma_{\omega}\left(A_{2}^{t=i \beta}, P\right)= & -i \int_{0}^{\beta} d s \tilde{\omega}\left(b(P) \tilde{\alpha}_{i s+i \beta} b\left(A_{2}\right)\right) \\
& +i \int_{0}^{\beta} d s \tilde{\omega}\left(\left[\tilde{\alpha}_{i s} b\left(A_{2}\right), b(P)\right]\right) \\
= & -i \int_{0}^{\beta} d s \tilde{\omega}\left(b(P) \tilde{\alpha}_{i s} b\left(A_{2}\right)\right) \\
= & -i \beta\left(b(P), b\left(A_{2}\right)\right)_{\sim},
\end{aligned}
$$

proving the theorem. 
On the basis of Theorem 3.5 one would guess that the perturbed equilibrium state given by formula (4.1) should be obtained from a limit theorem for the density matrices

$$
\lim _{n} e^{-\beta\left(H+\tilde{P}_{n}\right)} .
$$

In other words the perturbed equilibrium state is the central limit of the state perturbed by a local fluctuation. Actually this should also be proved, and the KMS-property, stable against the central limit as provided in [12], should also be stable under small but macroscopic perturbations of the type of fluctuations. In fact this is already proved for product states, say for mean field systems [14; Theorem 4.1] and there is no reason why it is not generally valid.

Clearly the above theorem settles the stationary states under the perturbed evolutions. The unperturbed state is not stationary for the perturbed time evolution. In physics (see e.g. [4]) one is interested in the time evolution of the fluctuations under the perturbed dynamics, one considers the following expectation value; the well-known response function:

$$
\lim _{n} \omega\left(\alpha_{t}^{\tilde{P}_{n}}\left(\tilde{A}_{n}\right)\right)=\tilde{\omega}\left(\tilde{\alpha}_{t}^{P}(b(A))\right)
$$

as a function of the time $t$. For that we have the following result.

Theorem 4.2. Under the same conditions as for Theorem 4.1 we have:

$$
\begin{aligned}
\frac{d}{d t} \tilde{\omega}\left(\tilde{\alpha}_{t}^{P} b(A)\right) & =\frac{d}{d t} \tilde{\omega}^{-P}\left(\tilde{\alpha}_{t} b(A)\right) \\
& =-i \tilde{\omega}\left(\left[\tilde{\alpha}_{t} b(A), b(P)\right]\right) \\
& =\beta \frac{d}{d t}\left(b(P), \tilde{\alpha}_{t} b(A)\right)_{\sim}
\end{aligned}
$$

for all $A \in \mathscr{K}_{\omega}^{\mathrm{Re}}$.

Proof. By Theorem 3.4 and by $\tilde{\omega} \circ \tilde{\alpha}_{t}=\tilde{\omega}$ :

$$
\tilde{\omega}\left(\tilde{\alpha}_{t}^{P} W(A)\right)=\tilde{\omega}(W(A)) e^{-i \sigma_{\omega}\left(P^{t}, \alpha_{t} A\right)} .
$$

But

$$
i \sigma_{\omega}\left(P^{t}, \alpha_{t} A\right)=\tilde{\omega}\left(\left[b(P), \int_{0}^{t} d s \tilde{\alpha}_{s} b(A)\right]\right) .
$$

Using

$$
W(A)=\exp i b(A),
$$

we get

$$
\frac{d}{d t} \tilde{\omega}\left(\tilde{\alpha}_{t}^{P} b(A)\right)=-i \tilde{\omega}\left(\left[\alpha_{t} b(A), b(P)\right]\right) .
$$

On the other hand by Theorem 4.1, one gets immediately

$$
\tilde{\omega}^{-P}\left(\tilde{\alpha}_{t} W(A)\right)=\tilde{\omega}(W(A)) e^{i \beta\left(b(P), \tilde{\alpha}_{t} b(A)\right) \sim},
$$


and hence

$$
\begin{aligned}
\frac{d}{d t} \tilde{\omega}^{-P}\left(\tilde{\alpha}_{t} b(A)\right) & =\beta \frac{d}{d t}\left(b(P), \tilde{\alpha}_{t} b(A)\right)_{\sim} \\
& =i \tilde{\omega}\left(\left[b(P), \tilde{\alpha}_{t} b(A)\right]\right) .
\end{aligned}
$$

For the last step, we used the relation [6, Theorem 2.5] easily extended to the field operators, because $\tilde{\omega}$ is quasi-free.

The first equality in the theorem expresses the duality between the perturbations of the equilibrium state and the perturbations of the equilibrium dynamics on the level of macroscopic fluctuations. The other equalities express the linearity of the full response function in the perturbation and its relation with the Duhamel two-point function. As in [14] one can also compute the relative entropy in terms of this function. The explicit formula is given by:

$$
S\left(\tilde{\omega}^{P} \mid \omega\right)=-\frac{\beta^{2}}{2}(b(P), b(P))_{\sim} .
$$

The rigorous proof of this formula however is somewhat more delicate than in [14] because the operator $b(P)$ is unbounded. We do not enter into the details here.

Finally, we remark that it follows from our rigorous analysis that the response at a reversible micro-system can be purely linear at the macroscopic level. Furthermore, the response theory is fully described in terms of a reversible dynamics, also on the macroscopic level, contrary to what is suggested in the physics literature (see [14]).

\section{References}

1. Haake, F.: Statistical treatment of open systems by generalized Master Equations. Springer Tracts in Modern Physics, Vol. 66. Berlin, Heidelberg, New York: Springer 1973

2. Davies, E. B.: Quantum theory of open systems. New York: Academic Press 1976

3. Kubo, T.: J. Phys. Soc. Jpn 12, 570 (1957)

4. Kubo, R., Toda, M., Hashitsume, N.: Statistical physics, II, nonequilibrium statistical mechanics. Berlin, Heidelberg, New York: Springer 1985

5. Mori, H.: Progr. Theor. Phys. 33, 423 (1965)

6. Naudts, J., Verbeure, A., Weder, R.: Commun. Math. Phys. 44, 87 (1975)

7. Verbeure, A., Weder, R.: Commun. Math. Phys. 44, 101 (1975)

8. Van Kampen, N. G.: Phys. Norv. 5, 279 (1971)

9. Naudts, J., Pule', J. V., Verbeure, A.: J. Stat, Phys. 21, 279 (1979)

10. Bratteli, O., Robinson, D. W.: Operator algebras and quantum statistical mechanics Vol. I, II. Berlin, Heidelberg, New York: Springer 1979/1981

11. Haag, R, Kadison, R. V., Kastler, D.: Commun. Math. Phys. 33, 1 (1973)

12. Goderis, D., Verbeure, A., Vets, P.: Dynamics of fluctuations for quantum Lattice Systems, Commun. Math. Phys. 128, 533-549 (1990)

13. Manuceau, J., Verbeure, A.; Commun. Math. Phys. 9, 293 (1968)

14. Goderis, D., Verbeure, A., Vets, P.: Prob. Th. Rel. Fields 82, 257 (1989)

Communicated by H. Araki 
